\title{
The inevitable and incidental caesarian myomectomy
}

\author{
Vinita Sarbhai* \\ Department of Obstetrics and Gynecology, Kasturba Hospital, Delhi, India
}

Received: 20 October 2019

Accepted: 19 November 2019

\author{
*Correspondence: \\ Dr. Vinita Sarbhai, \\ E-mail: vinitasarbhai@gmail.com
}

Copyright: $\odot$ the author(s), publisher and licensee Medip Academy. This is an open-access article distributed under the terms of the Creative Commons Attribution Non-Commercial License, which permits unrestricted non-commercial use, distribution, and reproduction in any medium, provided the original work is properly cited.

\begin{abstract}
The management of fibroid encountered during caesarean remains controversial. The traditional approach has always been, not to perform myomectomy, because of the fear of haemorrhage due to increased vascularity and uterine atonicity of pregnant uterus and increased morbidity. Caesarean section was performed in 27-year G2P1 in view of previous LSCS, short inter-conception period, frank leaking and poor bishop score. After delivery of baby a fibroid of $6 \mathrm{~cm}$ by $5 \mathrm{~cm}$ was seen jutting out of left side of incision line. Myomectomy was performed, without any complication, as it was not possible to approximate the incision line without that. No excessive hemorrhage or postoperative complications were encountered.
\end{abstract}

Keywords: Caesarian myomectomy, Fibroid, Gravid uterus, LSCS, Myoma

\section{INTRODUCTION}

Fibroid is the most common benign tumor of female genital tract occurring in the reproductive age group. Thus, it is the most commonly encountered tumor during caesarian section. The traditional approach has always been, not to perform myomectomy, because of the fear of haemorrhage due to increased vascularity and uterine atonicity of pregnant uterus and increased morbidity. Rather the recommended safer approach is to perform classical caesarian section, if it is encountered in the lower segment, which itself is associated with a lot of haemorrage. With recent advances in the medical and surgical interventions, it is now possible to perform myomectomy during caesarian. A few studies have suggested that caesarian myomectomy can be performed without any serious life-threatening complications. There is an added advantage of avoiding another surgery later on, thus decreasing the risk of anaesthesia complications, hospital stay, cost of another operation and reduced adhesions. It has also been postulated that uterus in the postpartum phase is better adapted physiologically to control hemorrhage. As contractions and retractions of muscle fibers occur, the blood vessels are closed. Also, the onset of vascular changes for clot formation in placental bed helps in stopping the bleeding. ${ }^{1}$

\section{CASE REPORT}

27-year-old, G2P1, un-booked patient was admitted in emergency labor room in the department of obstetrics and gynecology with 36 weeks and 4 days pregnancy, with previous LSCS and leaking per vaginal since 6 hours. There was no history of labor pains. The previous caesarian was performed 18 months back for fetal distress and no records were available. She had an old ultrasound report, which was performed at twelve weeks of gestation. That reported a hypo echoic lesion of size 80 $\mathrm{mm}$ by $68 \mathrm{~mm}$ in the anterior wall of uterus in the lower uterine segment suggestive of fibroid with 12 weeks live fetus and normal decidual reaction (Figure 1).

Her vitals were stable and on per abdominal examination, uterus was of 36 weeks size with single live fetus in longitudinal lie and cephalic presentation. On vaginal examination, cervix was closed; long, un-effaced and frank, clear, leaking per vaginal was observed. A decision of caesarian section was taken in view of previous LSCS, 
short inter-conception period, frank leaking and poor bishop score. Upon delivery of $3.5 \mathrm{~kg}$ female child as vertex, it was observed that the fibroid of $6 \mathrm{~cm}$ by $5 \mathrm{~cm}$ was jutting out from the left side of lower segment and the incision line could not be approximated unless the fibroid was removed. Hence a decision for myomectomy was taken after counseling about the risk of hemorrhage and possibility of hysterectomy. It was an intramural leiomyoma and the capsule was already incised during the caesarian incision over the lower segment (Figure 2).

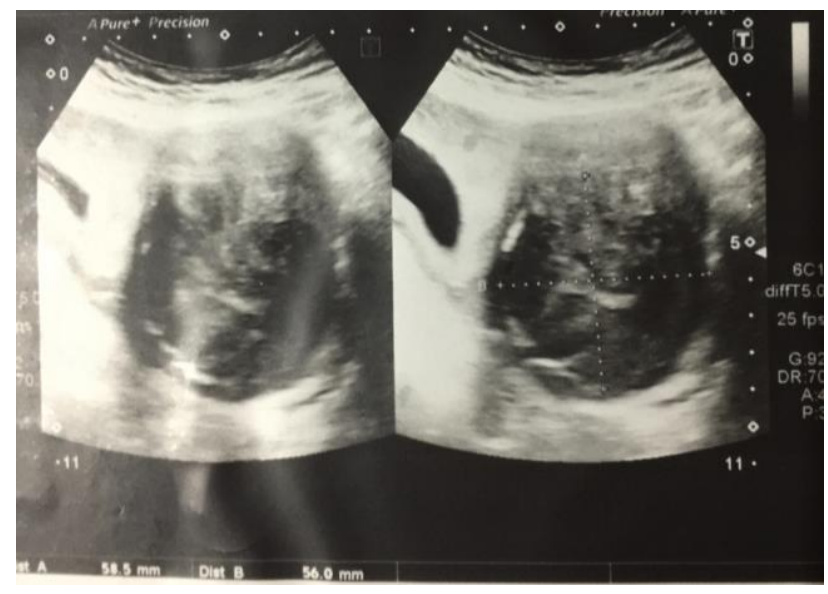

Figure 1: The ultrasound report at twelve weeks of gestation.

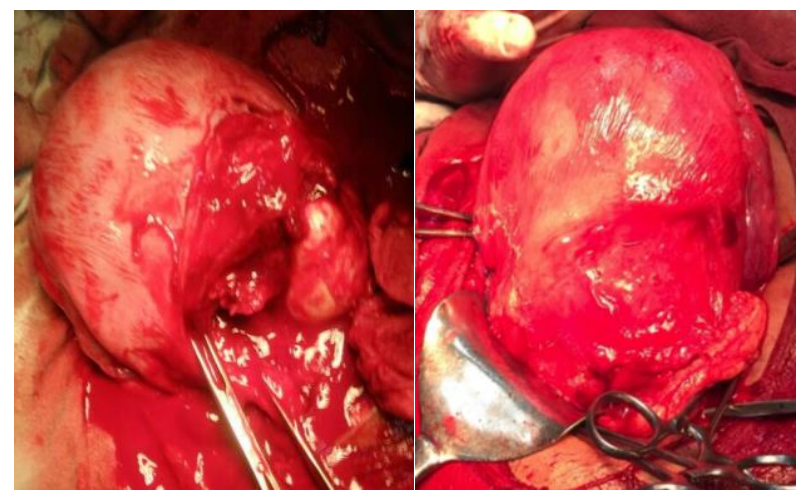

Figure 2: Pre-operative fibroid seen at incision line and uterus after myomectomy and closure of lower segment.

Myoma was fixed with Vulsellum forceps and enucleation carried out by a combination of blunt and sharp dissection. It was a soft fibroid with degenerative changes of pregnancy (Figure 3). The bleeding from the bed was secured with hemostatic sutures and electrocautery at places. The dead space was closed with number 1 Vicryl suture. Intravenous oxytocin drip and injection prostaglandin intramuscularly were given and the incision of caesarian was closed in two layers. Hemostasis was achieved and abdomen closed. The intraoperative blood loss was average and postoperative period was uneventful.

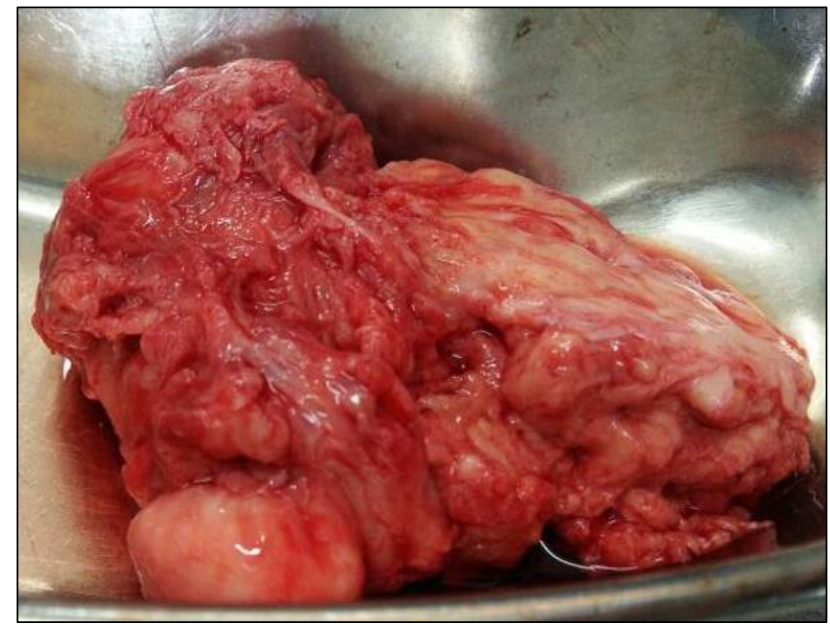

Figure 3: Degenerated fibroid after removal.

\section{DISCUSSION}

There is dilemma around the management of fibroid when encountered during caesarian and the traditional recommendation of not to perform myomectomy during caesarian section needs to be re-evaluated. Many authors have reported successful removal of fibroid without excessive hemorrhage or increased morbidity, especially if the fibroid is located in anterior wall, near the incision line as was seen in our patient.

In a retrospective cohort study, Roman AS et al, compared 111 women who underwent myomectomy at time of cesarean delivery and 257 women with documented fibroids during the index pregnancy who underwent cesarean delivery alone and reported that there was no statistically significant increase in the incidence of hemorrhage $(12.6 \%$ as compared with $12.8 \%$ in the control group), postpartum fever, operating time, and length of postpartum stay. ${ }^{2}$

In a series of 9 patients by Kant $\mathrm{A}$ et al, it was reported that cesarean myomectomy is not as dangerous as generations of obstetricians have been trained to believe. Enucleation of the fibroid is technically easier in gravid uterus owing to greater looseness of the capsule. ${ }^{1}$

Agarwal $\mathrm{K}$ et al, in a prospective study concluded that with the advent of better anesthesia and availability of blood, cesarean myomectomy is no longer a dreaded job in the hands of an experienced surgeon and in a wellequipped tertiary institution. ${ }^{3}$

In a feasibility and safety study by Lovina SM, caesarian myomectomy was carried out in 8 patients and it was concluded that patient selection is crucial in Caesarean myomectomy. ${ }^{4}$ Large fundal intramural fibroids should be intuitively avoided. Measures to minimize blood loss, like preoperative placement of uterine artery, balloon catheters, utero-tonic drugs, uterine artery ligation, uterine tourniquets, stepwise de-vascularization, and 
post-Caesarean uterine artery embolization would optimize outcomes and significantly decrease the chance of hysterectomy.

Awoleke JO et al, stated the advantages of concurrent myomectomy to be prevention of subsequent separate surgery, minimization of puerperal uterine sub-involution and other complications like menorrhagia, anemia, and pain. $^{5}$

\section{CONCLUSION}

In conclusion caesarean myomectomy appears to be a safe procedure if carried out in properly selected patients, with good surgical technique. Guidelines should be formulated after larger multi-centric randomized casecontrol trials for patient selection, proper surgical technique and haemostatic measures.

Funding: No funding sources

Conflict of interest: None declared

Ethical approval: No required

\section{REFERENCES}

1. Kant A, Manuja S, Pandey R. Cesarean myomectomy. The $\mathrm{J}$ Obstet Gynecol India. 2007;57(2):128-30.

2. Roman AS, Tabsh KMA. Myomectomy at time of cesarean delivery; a retrospective cohort study. BMC Preg Child Birth. 2004;4:14-7.

3. Agarwal K, Agarwal L, Agarwal A. Caesarean myomectomy: prospective study. NJIRM. 2011;2(3):11-4.

4. Machado LS, Gowri V, Al-Riyami N, Al-Kharusi L. Caesarean myomectomy: feasibility and safety. Sultan Qaboos University Med J. 2012;12(2):190-6.

5. Awoleke JO. Myomectomy during caesarean birth in fibroid-endemic, low-resource settings. Obstet Gynecol Int. 2013;520834:1-6.

Cite this article as: Sarbhai V. The inevitable and incidental caesarian myomectomy. Int J Reprod Contracept Obstet Gynecol 2020;9:421-3. 